\title{
Estimates of contraceptive failure from the 2002 National Survey of Family Growth
}

\author{
Kathryn Kost ${ }^{1}$, Susheela Singh ${ }^{1}$, Barbara Vaughan ${ }^{1}$, James Trussell ${ }^{2}$, and Akinrinola \\ Bankole $^{1}$
}

${ }^{1}$ Guttmacher Institute, 125 Maiden Lane, 7th floor, New York, NY 10038, USA. ${ }^{2}$ Office of Population Research, Wallace Hall, Princeton University, Princeton, NJ 08544, USA and The Hull-York Medical School, Hertford Building, University of Hull, Cottingham Road, Hull HU6 7RX, England.

\begin{abstract}
Background-In 2001, the U.S. government's Healthy People 2010 initiative set a goal of reducing contraceptive failure during the first year of use from $13 \%$ in 1995 to $7 \%$ by 2010 . We provide updated estimates of contraceptive failure for the most commonly used reversible methods in the United States, as well as an assessment of changes in failure rates from 1995 to 2002.

Study design-Estimates are obtained using the 2002 National Survey of Family Growth (NSFG), a nationally representative sample of U.S. women containing information on their characteristics, pregnancies, and contraceptive use. We also use the 2001 Abortion Patient Survey to correct for underreporting of abortion in the NSFG. We measure trends in contraceptive failure between 1995 and 2002, provide new estimates for several population subgroups, examine changes in subgroup differences since 1995, and identify socioeconomic characteristics associated with elevated risks of failure for three commonly used reversible contraceptive methods in the U.S.: the pill, male condom and withdrawal.
\end{abstract}

Results - In 2002, 12.4\% of all episodes of contraceptive use ended with a failure within 12 months after initiation of use. Injectable and oral contraceptives remain the most effective reversible methods used by women in the U.S., with probabilities of failure during the first 12 months of use of $7 \%$ and $9 \%$, respectively. The probabilities of failure for withdrawal (18\%) and the condom (17\%) are similar. Reliance on fertility-awareness-based methods results in the highest probability of failure (25\%). Population subgroups experience different probabilities of failure, but the characteristics of users that may predict elevated risks are not the same for all methods.

Conclusion-There was no clear improvement in contraceptive effectiveness between 1995 and 2002. Failure rates remain high for users of the condom, withdrawal and fertility-awareness methods, but for all methods, the risk of failure is greatly affected by socioeconomic characteristics of the users.

\footnotetext{
(C) 2009 Elsevier Inc. All rights reserved.

Corresponding author, Kathryn Kost, 125 Maiden Lane, 7th floor New York, NY 10038 USA, Tel: 212248 1111, Fax: 2122481951 kkost@guttmacher.org.

Publisher's Disclaimer: This is a PDF file of an unedited manuscript that has been accepted for publication. As a service to our customers we are providing this early version of the manuscript. The manuscript will undergo copyediting, typesetting, and review of the resulting proof before it is published in its final citable form. Please note that during the production process errors may be discovered which could affect the content, and all legal disclaimers that apply to the journal pertain.
} 


\section{Keywords}

Contraception; Contraceptive Methods; Contraceptive Effectiveness; Contraceptive Failure; National Survey of Family Growth; NSFG

\section{Introduction}

Contraceptive failure is a primary cause of unintended pregnancy in the United States. Nearly half of all pregnancies are unintended, and nearly half of the 3.1 million unintended pregnancies in 2001 occurred to women who were using contraception [1]. Thus, reducing the risk of failure during contraceptive use would have a major impact on reducing unintended pregnancy in the United States.

The most recent estimates of contraceptive failure are for the mid-1990s [2-4]. These estimates have been essential for informing women's contraceptive decisions in the United States, as well as assisting providers in counseling, providing the most current information for teaching of sex education, and informing the general public. However, these estimates are now over 10 years old and may not be an accurate reflection of American women's current experience in using reversible contraceptive methods. This paper provides updated information on contraceptive failure using data from the most recent National Survey of Family Growth (NSFG), carried out in 2002. It also permits an assessment of trends in contraceptive failure between 1995 and 2002.

In January 2000, the U.S. Department of Health and Human Services released the "Healthy People 2010" initiative, setting national goals for improvement in a broad set of health indicators of the U.S. population, including the reduction of unintended pregnancy and of contraceptive failure. In particular, the initiative called for a reduction in the proportion of women becoming pregnant during the first year of use of a reversible contraceptive from 13\% (as measured in 1995) to 7\% by 2010 [5]. However, studies prior to 2000 did not find any noticeable improvements in contraceptive effectiveness from the 1980s to the early 1990s [2, 4,6]. It is crucial then for policy makers to know if the effectiveness with which American women use specific contraceptives is improving, showing no change or declining. Information on the trend in contraceptive effectiveness since 1995 will contribute to decisions about the level of resources that may be needed to promote higher levels of effectiveness. Understanding trends can also inform service providers' decisions about the content of information and services offered to users in order to improve an individual client's chance of success with a particular method. It is also very important for service providers to have information on differences in contraceptive effectiveness among key population subgroups. Such information helps providers tailor counseling to the particular groups they serve.

Estimates of contraceptive effectiveness from a population-based survey capture the probability of failure during "typical" use of the method, including imperfect use. Therefore, these are not measures of the inherent efficacy of a contraceptive method when used perfectly (correctly and consistently). In contrast, clinical trials provide the best estimates of the probability of failure during "perfect" use of a method and are very useful as a standard against which typical or population-based estimates may be compared. However, typical-use estimates of contraceptive failure based on women's behavior reported in population surveys are necessary for monitoring the actual experience of average contraceptive users, given their wideranging circumstances, characteristics, motivation and attitudes.

Population-based surveys such as the NSFG have some disadvantages, particularly the high level of underreporting of induced abortion; to obtain an accurate reflection of women's 
pregnancy experiences, the data must be adjusted to account for the discrepancy between reporting and actual occurrence of induced abortion. Our study uses an existing analytical approach that was developed in the 1980s to adjust for unreported abortions [6]. We use information from the 2000/2001 Abortion Patient Survey, combined with existing national data on the total number of abortions in the United States, to adjust for underreporting of contraceptive failures resolved by abortion in the NSFG.

We compare first-year probabilities of contraceptive failure estimated from the 1995 NSFG with newly calculated estimates from the 2002 NSFG. We also provide new estimates of contraceptive failure for several key population subgroups in the U.S. - segmented by age, parity, union status, race-ethnicity and poverty status — as well as examine changes in subgroup differences since 1995 . Finally, we identify socioeconomic characteristics associated with elevated risks of failure for the three commonly used reversible contraceptives in the U.S.: the pill, male condom and withdrawal.

\section{Data and methods}

\subsection{National Survey of Family Growth}

Nationally representative information on contraceptive use and pregnancies was obtained from Round 6 of the National Survey of Family Growth (NSFG), conducted by the National Center for Health Statistics (NCHS) in 2002-2003. The NSFG contains a sample of 7,643 women, ages 15-44 and includes extensive information on the respondents' demographic and socioeconomic characteristics, their pregnancy and union status histories, and a detailed monthby-month contraceptive use calendar from January 1999 to the date of interview (median = September 2002). Using these data, we constructed a new data file consisting of the intervals of contraceptive use observed during the roughly 33/4-year period covered by the contraceptive use calendar These intervals or "segments" of contraceptive use constitute our unit of analysis for the measurement of contraceptive failure (see Appendix: Segment Data).

\subsection{Correcting for underreporting of abortions in the NSFG}

The most accurate estimate of the number of abortions that occur each year in the United States is calculated periodically by the Guttmacher Institute, through national surveys of all known abortion providers in the U.S. [7]. ${ }^{\mathrm{a}}$ We know from previous analyses that only $47 \%$ of the 6.5 million abortions that occurred during the five years preceding the 2002 NSFG were reported by the survey respondents [8]. Because at least half of the pregnancies terminated by induced abortions occurred during use of contraception [9], estimates of failure relying only on NSFG data are likely to be underestimated by the omission of abortions by NSFG respondents. In addition, characteristics of women that are associated with higher or lower rates of abortion underreporting are also associated with the probability of contraceptive failure $[2-4,6,8,10$, $11]$, so that underreporting can also lead to misleading differentials in the risk of failure among subgroups.

To correct the number of abortions resulting from contraceptive failure in the NSFG, we first calculated the total number of abortions that occurred in the United States for the time period covered in our analysis. The corrected numbers of abortions were then distributed among the appropriate population subgroups by employing the 2000/2001 Abortion Patient Survey (APS), a nationally representative sample of all abortion patients, including information on contraceptive use at the time of pregnancy as well as socioeconomic and demographic characteristics of women.

${ }^{\mathrm{a} D a t a}$ on the number of abortions that occur in the US are nearly complete, and are estimated to be within $3-4 \%$ of the actual number [7]. 
We corrected the number of abortions in the NSFG contraceptive segment data set by adding APS abortions resulting from contraceptive failure. The abortions obtained using the national counts and the APS survey represent all abortions occurring in the U.S. during our period of analysis. Therefore, the abortions reported in the NSFG were dropped from the analysis so that only the APS abortions are included. ${ }^{\mathrm{b}}$ To obtain the appropriate numbers of abortions and to distribute the abortions among the correct socioeconomic groups, we weighted the APS sample up to the national number of abortions for the same time period covered by the contraceptive calendar in the NSFG (January 1999 to September 2002). The population-scaled weights were calculated from the distribution of all abortions during the observation period by age and race, and the corresponding unweighted APS observations were multiplied by these weights. ${ }^{\mathrm{c}}$

This correction is done at the aggregate level, for population subgroups, because it would be impossible to allocate the abortions from the APS to individual contraceptive use segments in the NSFG. The total count of failures resulting in abortion for each subgroup replaces the NSFG count for that group. ${ }^{\mathrm{d}}$

\subsection{Analysis of trends}

An important advantage of the approach and methodology applied in the present analysis and of the use of population-based surveys such as the NSFG is that the estimates are comparable over time and permit examination of trends in contraceptive failure. Because of the consistency in the design of the 1995 and 2002 NSFG surveys and in the abortion patient surveys of 1994 and 2001, we are able to apply a nearly identical methodology to the analysis, eliminating one potentially large cause of incomparability in results obtained across surveys and time. To ensure that results are as closely comparable as possible across the two time periods, estimates for 1995 were re-calculated using the same methodology that we use for the 2002 analysis.

\subsection{Variables in the analyses}

We provide estimates of the probability of failure for the five most commonly used methods in the United States: injectable contraceptives", oral contraceptives ("the pill"), male condoms, fertility-awareness-based methods ("rhythm-", "calendar-", "mucus-", and "temperature-" methods, "periodic abstinence" or "natural family planning"), and withdrawal. Other methods used by women include the IUD, implant, cervical cap, female condom, sponge, jelly, cream, foam, suppositories, contraceptive film, and other spermicides. ${ }^{\mathrm{f}}$ However, there were not enough uses of these other methods in the NSFG data to allow separate estimates of contraceptive failure. Even after combining all barrier and spermicide methods other than the male condom into a single group, there was still an insufficient number of observations for

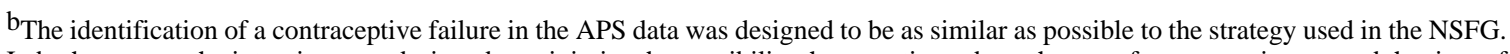
In both surveys, the interview was designed to minimize the possibility that questions about the use of contraception around the time of pregnancy would encourage women to attribute the conception to contraceptive failure. However, unlike the NSFG, contraceptive use in the APS is not recorded in a calendar. Instead, women are asked about the last method used before they became pregnant and whether they had stopped using contraception before they became pregnant. The latter question is similar to the one asked in the NSFG when a woman is using contraception in the month of conception and was therefore used to identify failures.

${ }^{\mathrm{c}}$ Eighteen weighting factors were calculated for each combination of six age groups (<20, 20-24, 25-29, 30-34, 35-39 and 40+) and three race groups (blacks, whites and all others).

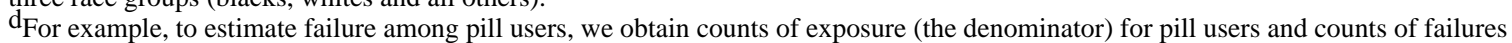
(numerator) for that group. In the numerator, APS pill failures are substituted for any NSFG pill failures resulting in abortion. The denominators are not adjusted because it is assumed that reports of the duration of contraceptive use in the NSFG are reasonably correct. However, some women who experienced a failure may have reported the abortion as a miscarriage, which means that, with the addition of the APS abortion, the failure could be counted twice in the numerator with the exposure counted only once in the denominator, and this could lead to an overestimate of the probability of failure. However, there is very little evidence that this type of misreporting is common either in the 2002 NSFG or in past surveys [8,10-11].

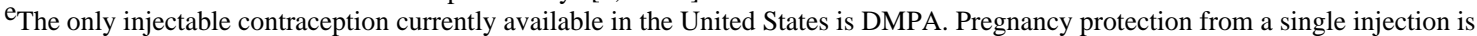
expected to last 12 to 14 weeks.

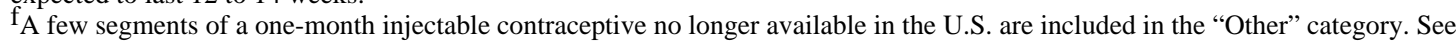
Appendix: Segments of Use.
} 
analysis. We required a minimum of 50 segments at every month during the first year of use to calculate a probability of failure for an individual method; all other methods that did not meet this criterion are grouped in an "other" category, and although estimates are not presented for this residual group, segments of use of these methods are included in estimates for all reversible methods combined.

Prior research has shown that the risk of contraceptive failure is affected by how long a method has been used and by socioeconomic and demographic characteristics of the user [2-4,6]. We therefore examine the probability of failure by duration of use, age, race and ethnicity, union status, poverty status, parity and pregnancy intention status.

Age of the woman is measured at the start of each segment of contraceptive use and is grouped into four categories: $15-19,20-24,25-29$ and 30+. We use three categories for race and ethnicity: Hispanic, non-Hispanic black, and non-Hispanic white or other. There were too few observations for "other" race and ethnic groups to sustain separate estimates.

The woman's union status - a measure that combines both her formal marital status and whether she was living with a non-spouse partner (cohabitation) — is defined at the start of each segment (see Appendix: Segment Data). There are four union status groups: married, cohabiting, formerly married, and never married.

Poverty status is measured as the ratio of the woman's household income in the year prior to interview to the federal poverty standard given her family size. Women whose household income is less than $100 \%$ of the poverty standard are considered to be poor or "in poverty". Those at 100 to $199 \%$ of the poverty standard are considered near poor and those at $200+\%$ are relatively well off.g

Parity is coded into three categories: no previous births, one previous birth and two or more previous births. In some of our analyses, we found that the crucial distinction was between women who have had a birth and those who have not; for these, parity is coded into two categories: no previous births and one or more previous births.

Intention status is included in our analyses to capture the woman's intentions toward having a birth or another birth while she was using contraception. Two categories are used, one to include women who did not intend a birth and the other to include both those who did intend a birth at some time in the future and those who were unsure of their intentions. ${ }^{\text {h }}$

\subsection{Statistical methods}

For probabilities of failure by method used and for probabilities of failure using any method by socioeconomic characteristics of the woman, we analyze segments of use using a life table to estimate the associated single-decrement probability of failure by duration. Women who were already using a method of contraception at the beginning of the calendar observation period entered the life table at the duration of use at that time. If a woman discontinued use of

\footnotetext{
gIt is possible that a woman who is found to be in poverty at interview was not so at an earlier date, even just 1 or 2 years earlier, particularly if she had one or more births during the calendar period. The reason is that poverty status is partially determined by - and can be greatly affected by — number of children. Our assumption means that we may dilute any effect of being in poverty, producing a conservative estimate of its effects.

hThe woman's intention status at the start of an interval ending in pregnancy — a "closed" interval — is taken from the answer to the question, "Right before you got pregnant... did you yourself want to have a(another) baby at any time in the future?" (asked during the pregnancy history interview). The question refers to the first pregnancy immediately following the segment of use. This question was not asked in closed intervals if the woman said she had stopped using (or was not using) contraception because she wanted to get pregnant as soon as possible. In that case, the woman's intention status is assumed to be "wanted to have a baby". If there are no pregnancies between the end of the segment and the interview — an "open" interval — the intention status is measured as of the date of the interview, based on the question, "Do you intend to have (more) children in the future?"
} 
a method for reasons other than failure, we considered this to be a competing risk; we estimate the gross probability of failure in the absence of competing risks using the exact formula developed by Trussell and Menken [12]. Women who were still using a method of contraception at the cutoff point were censored at the duration of use at that time. The life table provides estimates of the probability of failure for each month of use; these can be cumulated to estimate the probability of failure up to a given duration of use, typically presented at 3,6, and 12 months. ${ }^{i}$

All analyses for this article were conducted using the abortion-corrected data. However, for the method-specific probabilities of failure within 3, 6 and 12 months of use, we compare estimates from the 2002 NSFG using data both uncorrected and corrected for abortion underreporting, in order to examine the effects of the correction procedure.

All comparisons to the 1995 survey required reanalysis of the 1995 data. The probabilities of failure published in previous analyses of the 1995 NSFG were based on unweighted data and were obtained from a multivariate model rather than a life table. For our comparisons, we reanalyzed the 1995 data using weights and the identical life table analysis we used for the 2002 data.

We used a method devised by Peto and colleagues [13] to calculate 95\% confidence intervals surrounding the estimated probabilities. This method produces more conservative confidence intervals than the more traditionally used Greenwood formula. However, we combine two data sources - the NSFG and the APS surveys - to obtain abortion-corrected estimates. We reason that the Peto estimates are designed to reflect increased uncertainty caused by censoring at durations when no failures are observed (i.e., when the Greenwood confidence intervals do not change). Although there are very few such instances in our data, the conservative nature of the Peto estimates is nevertheless appropriate given the increased level of uncertainty caused by the combination of the two data sources.

We calculated method-specific probabilities of failure within 12 months of initiating use using abortion-corrected data for both the 1995 and 2002 NSFG data sets. The statistical significance of any changes in probabilities of failure between the two time periods covered in these surveys is examined using standard z-tests. The 1995 estimates are obtained from the contraceptive and reproductive experiences of women from January 1991 through mid-year 1995, while the 2002 estimates refer to the time period from January 1999 to mid-year 2002.

We also conducted three separate multivariate analyses of the probability of failure during use of the pill, condom and withdrawal. ${ }^{j}$ In each analysis, we examine whether the risk of failure varied by characteristics of the users. We used unweighted, abortion corrected data and employed the GENMOD procedure in the SAS statistical analysis package, which allows us to fit a generalized linear model to count data with the assumption of a Poisson distribution, and thereby to estimate the relative risk of failure associated with each socioeconomic characteristic in a multivariate context [14].

While we use weighted data for our analyses of the overall probabilities of failure by method and for individual subgroups, we use unweighted data for the multivariate analyses of the factors affecting failure for specific methods in order to employ standard statistical tests of

\footnotetext{
${ }^{i}$ A probability of failure within the first 12 months of use includes all women who initiated a segment of use within the observation period, regardless of whether they had used the method previously. In other words, "the first 12 months of use" does not mean within the first 12 months that the woman has ever used the method. Segments from women who are returning to use of a method they have used many times before are included with segments from women who are using the method for the first time.

$\mathrm{j}_{\mathrm{We}}$ required at least 800 segments for inclusion in multivariate analyses. Only the pill $(\mathrm{n}=2,541)$, condom $(\mathrm{n}=3,845)$, and withdrawal $(\mathrm{n}=848)$ met that requirement.
} 
model fit. ${ }^{\mathrm{k}}$ The weights in the NSFG reflect the sampling design, including differential sampling by age and race [15]. We control for this differential sampling by including both age and race in all multivariate models. ${ }^{1}$ We also compared the results from our final models with those produced using weighted data, as a check for uncontrolled sample composition effects in the estimates.

\section{Results}

\subsection{Probabilities of contraceptive failure in 2002}

Within 3 months of the initiation of use of a reversible method of contraception, $4.2 \%$ of all women experienced a contraceptive failure (Table 1). At 6 months, $7.3 \%$ had experienced a failure, and by 12 months of use $12.4 \%$ had experienced a contraceptive failure.

Injectable contraceptives and the pill remain the most effective reversible methods used by women in the U.S. and have similar probabilities of failure in the first 3 months of use. However, by the $6^{\text {th }}$ and $12^{\text {th }}$ month of use, injectables may be more effective than the pill; there is a difference of about two percentage points in the probability of failure at both durations, although it is not statistically significant.

The 12-month probability of failure is significantly higher for the male condom than for injectables and the pill (17\%, 7\% and 9\%, respectively). The estimated 12-month probability of failure for withdrawal is almost the same as that for the condom (18\%). Reliance on fertilityawareness-based methods results in the highest probability of failure, as $25 \%$ will become pregnant within 12 months of initiating use. The probability of failure for fertility-awarenessbased methods is substantially higher than that for the condom and withdrawal at all durations of use, although again the differences are not statistically significant.

Table 1 also shows the effects of correcting for unreported abortions on the estimates of failure. There is virtually no difference in the estimates for the first 3 months of use (4.2\%, corrected and $4.1 \%$, uncorrected), and the first 6 months of use (7.3\%, corrected and 7.1\%, uncorrected). But the inclusion of unreported abortions leads to a slightly higher estimated probability of failure in the first 12 months of use (12.4\%, corrected and $11.1 \%$, uncorrected).

The effect of the correction for abortion varies for estimates of the individual methods.

Inclusion of unreported abortions has very little effect on the estimate of failure for withdrawal; the probability of failure within 12 months of use is $18.8 \%$, uncorrected for abortion reporting, and $18.4 \%$, corrected. The correction leads to a slightly lower estimate for injectables $(7.5 \%$ uncorrected, and $6.7 \%$ corrected) and a slightly higher estimate for the pill (7.7\% uncorrected, and $8.7 \%$ corrected). But the correction increases the estimate for fertility-awareness-based methods ( $23.0 \%$ uncorrected, and $25.3 \%$ corrected) and leads to a much higher estimate for condoms (13.9\% uncorrected, and $17.4 \%$ corrected).

\subsection{Trends in Contraceptive Failure, 1995-2002}

Reanalysis of the 1995 data produced somewhat higher estimates of failure than previously published [2]. Even so, there was no clear improvement in contraceptive effectiveness between 1995 and 2002. However, the probability of contraceptive failure during use of all reversible

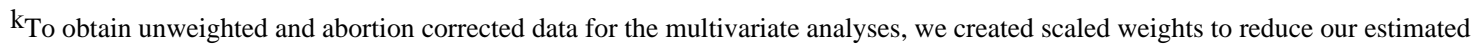
population numbers of abortions back to unweighted counts for the time period covered by the NSFG. The population counts of abortion were scaled down to unweighted numbers by multiplying the counts of APS abortions in each cell by a scale factor calculated as the ratio of unweighted to weighted exposure in the cell, obtained from the NSFG segments.

${ }^{1}$ If the inclusion of either age or race/ethnicity did not have a significant effect on the model fit or on the effects of the other variables, the variable was dropped in the final stages of the model selection process.
} 
contraceptive methods did decline by 2.5 percentage points, from $14.9 \%$ to $12.4 \%$; while this change is not statistically significant, it is substantial (Table 2). Similarly, method-specific differences are evident, though none is statistically significant. Estimates of failure for injectables and fertility-awareness-based methods are higher in 2002 than in 1995, while the probability of failure for withdrawal dropped from $28.4 \%$ in 1995 to $18.4 \%$ in 2002 . There was very little change in the probabilities of failure for the pill and condom between 1995 and 2002. $\mathrm{m}$

\subsection{Subgroup differences in contraceptive failure, 1995 and 2002}

In both 1995 and 2002, women aged 30 and older were less likely to experience a failure than were younger women (Table 3). In 2002, this age difference appears to have increased, as women over age 30 were significantly less likely to experience a contraceptive failure than were younger age groups. This age group also experienced a larger improvement between 1995 and 2002 than did younger age groups.

Both in 1995 and in 2002, non-Hispanic black women were significantly more likely to experience a contraceptive failure than non-Hispanic women of white/other races. NonHispanic black women experienced a failure in the first year of use at roughly twice the rate for non-Hispanic women of white/other races (in 2002, the estimated first-year probabilities were $21.3 \%$ and $10.1 \%$, respectively). Hispanic women also faced a higher risk of failure in $2002(15.0 \%)$ compared with non-Hispanic women of white/other races (10.1\%), although the difference was not statistically significant ( $\mathrm{p}$-value $=.08$ ). Declines in the probability of failure among all three race and ethnic groups between 1995 and 2002 were not statistically significant, although the absolute changes were not trivial (2-3 percentage points; Table 3).

In 1995, there was virtually no difference in the probability of failure for women who did not intend to have a birth (or another birth) and those who did (14.3\% and $15.1 \%$, respectively). But by 2002 , women who did not intend to have a birth were significantly less likely to experience a contraceptive failure $(9.2 \%)$ than women who did want a birth sometime in the future or were not sure about their intentions (13.9\%).

Similarly, in 1995, women who had not had a birth were no more or less likely to experience a failure than women who had one or more births. But in 2002, those who had no previous births were far less likely to experience a failure (6.2\%) than those who had had one previous birth $(18.0 \%)$ or those who had had two or more $(16.3 \%)$.

The risk of failure among the poorest women (those below the poverty threshold of 100\%) remains high in 2002 at $19.9 \%$, although it has decreased from a much higher level in 1995, $25.5 \%(\mathrm{p}=.09)$. A decrease is also evident among those in the highest income status group (at $200 \%$ or more of the poverty threshold; from $11.6 \%$ to $8.4 \%$ ). There was no change between 1995 and 2002 in the risk of failure among women in the middle poverty-status category, those at 100-199\% of poverty. But in both 1995 and 2002, the poorest women had a significantly higher probability of experiencing contraceptive failure than did better off women $(\geq 200 \%$ of the poverty level). And by 2002, the gap between women in the highest poverty status group ( $\geq 200 \%$ of the poverty level) and all other women had widened, suggesting that the probability of failure continues to be strongly affected by family incomes.

Finally, the risk of failure is affected by the woman's union status. In 1995, all unmarried women - whether cohabiting, formerly married or never married - had a significantly higher

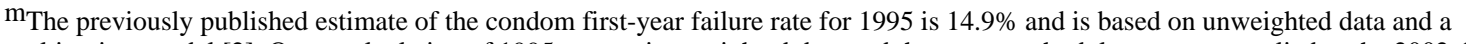
multivariate model [2]. Our recalculation of 1995 rates using weighted data and the same methodology as was applied to the 2002 data resulted in an increased estimate of $17.8 \%$.
} 
risk of failure than married women. By 2002, only cohabiting women had a significantly higher risk of failure than married women. Women in cohabiting union had the highest risk of failure in both 1995 (28.7\%) and 2002 (21.7\%), while married women had the lowest (10.4\% in 1995; $9.5 \%$ in 2002). In 2002, women who were not married and not living with a partner had an intermediate risk, with $11.5 \%$ of never married women and $12.2 \%$ of formerly married women experiencing a failure in the first year of use. However, in 1995, formerly married women were nearly as likely to experience a failure as cohabiting women; in fact, the difference in the estimates is not statistically significant (not shown, p-value=.27). But by 2002 , formerly married women had a significantly lower risk of failure than cohabiting women $(12.2 \%$ and $21.7 \%$, respectively; $\mathrm{p}$-value $=.08$ ), and formerly married women were no more likely to experience a failure than currently married women ( $\mathrm{p}$-value $=.33$ ).

\subsection{Determinants of failure during use of the pill, condom and withdrawal}

Table 4 shows the results of the multivariate analyses in which we examined the socioeconomic characteristics associated with failure for three specific methods. In the analysis for the pill, we combined the age groups 20-24 and 25-29 as we found no difference in the probability of failure between the two groups. The woman's age, her intention toward a future birth, whether or not she already had a child, and her union status were the most important determinants of pill failure. Once these factors were taken into account, her duration of use, race/ethnicity and poverty status had no effect on the risk of a pill failure. ${ }^{\mathrm{n}}$ Relative risks for the variables in the final model are shown in Table 4.

Pill users age 30 or older have a significantly lower risk of experiencing a failure than that for any other age group, after controlling for other factors. Teens are more than twice as likely to experience a pill failure as women age 30 or older, while women in their 20 s are more than 1.7 times as likely to get pregnant while using the pill as older women.

Women using the pill to avoid pregnancy but who intend to have a birth at some time in the future are 1.7 times as likely to experience failure as those not intending to have a future birth. Single and cohabiting women are more likely to experience a pill failure than are currently married women. Cohabiting women are $2 \frac{1}{2}$ times as likely to get pregnant while using the pill, and women who are not in a union are almost twice as likely to become pregnant as are currently married pill users.

Pill users who have ever had a birth are far more likely to have a failure than those who have never had a birth. However, parity partially reflects how well the woman has used contraception in the past (i.e., no births means she successfully avoided pregnancy) as well as fecundity, so that nulliparous women are more likely to include those who are sub-fecund.

In the analysis of condom failure, we combined categories in several variables because there was no difference in the risk of failure among the more disaggregated groups. Age was collapsed into two groups, condom users less than age 30 and those ages 30 or older. Race/ ethnicity was also collapsed into two groups, non-Hispanic black women and all others (Hispanic, white, and others). As in the analysis of pill failure, the woman's age, intention status, union status and parity played a major role in the risk of failure while duration of use had no effect on the risk. However, unlike for the pill, both race/ethnicity and poverty also affect the risk of condom failure.

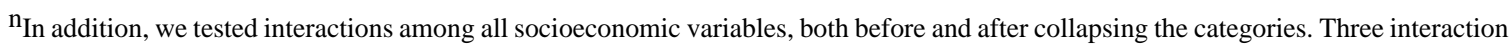
effects were found to significantly improve the fit of the model: age by intention status, age by union status, and one 3-way interaction: age by intention by union status. However, we did not find that the inclusion of the interaction terms added substantively to the models and chose to present the models with main effects only.
} 
Women age 30 and older enjoy the lowest risk of condom failure, as do women who do not intend more births and those who are not in a cohabiting union (that is, either single or currently married; Table 4). Like pill users, women who rely on the condom and have had at least one birth are more likely to experience a contraceptive failure than those who have never had a birth ( 2.5 times as likely). And black women who rely on the condom are about $63 \%$ more likely to experience a failure than all other women. Poor and low-income women are almost twice as likely to experience a condom failure as are women economically better off.

In the analysis of failure during use of withdrawal, we found that the risks among formerly and currently married women were similar; we therefore created a dichotomous union status variable using the categories ever-married and never-married. The significant predictors of failure for withdrawal are intention status, parity, poverty status, and union status. Once these factors were taken into account, duration of use, age and race/ethnicity had no effect on the risk of a failure.

Women who intend to have future births or are unsure of their intentions are roughly 3 times as likely to become pregnant while using withdrawal as those who do not intend a future birth (Table 4). And withdrawal users who have never been married are more than twice as likely to experience a failure as users who have ever been married. As during use of the pill and condom, nulliparous withdrawal users are about a quarter as likely to experience a failure as those who have had a birth. And, women living below or near poverty and relying on the withdrawal method experience almost twice the risk of failure as women whose family incomes are at least double the poverty threshold.

\section{Discussion}

A high proportion of all American women who are at risk of unintended pregnancy use reversible methods of contraception (57\%) and the majority of women currently using reversible methods use the pill or the condom $(76 \%) .{ }^{\circ}$ Our new national estimates show that about one in every eight uses of a reversible method results in a contraceptive failure during the first year. This estimate reflects typical use, including incorrect and inconsistent use. Between 1995 and 2002, the probability of contraceptive failure during use of all reversible methods combined declined by almost $20 \%$-from $14.9 \%$ to $12.4 \%$-although the change is not statistically significant. Increased proportions using two of the more effective methods (injectables and the pill), and a lower proportion using the condom alone (which has much lower effectiveness than the two hormonal methods) account for part of this overall improvement in contraceptive effectiveness.

Our estimates show a high probability of failure for fertility-awareness-based methods (25\%), and an intermediate risk for the male condom and withdrawal (17\% and $18 \%$, respectively). Two hormonal methods, injectables and the pill, (with probabilities of failure of $7 \%$ and $9 \%$, respectively) are by far the most effective reversible methods being used by a sizable number of U.S. women.p

The effect of the correction for abortion varies for estimates of the individual methods. This variation could be a reflection of differences in the propensity to report abortions among users of the various methods in the NSFG or a differential tendency to report method use at the time of conception in the two surveys. Inclusion of unreported abortions has very little effect on the estimate of failure for withdrawal, leads to a slightly lower estimate for injectables and a slightly

\footnotetext{
o"Use of contraception" is based on women's reports of use in the month of the NSFG interview. The proportion using these methods is calculated from Tables 5 and 6, in Mosher, et al. (2004) [16].

p This study does not provide information on the newer hormonal methods such as the patch, vaginal ring, and single rod implant because these methods were either not marketed or not yet widely used at the time covered by the 2002 NSFG contraceptive use calendar.
} 
higher estimate for the pill. But the correction increases the estimate for fertility-awarenessbased methods and leads to a much higher estimate for condoms. The effect of the correction implies that more abortions are reported during use of fertility-awareness-based methods and the condom in the APS than in the NSFG.

Changes in the characteristics of users of specific methods may help to explain changes in the probability of contraceptive failure between 1995 and 2002. For example, socioeconomic groups with traditionally higher risks of failure may make up a greater proportion of users of certain methods in 2002 compared to 1995, and thereby account for a rise in estimates of the probability of contraceptive failure. An increase in the first year probability of failure for the injectable contraceptive occurred between 1995 and 2002 (from 5.4\% to 6.7\%), and while this increase was not statistically significant, it may be due in part to a shift in the composition of the population of women using it. In fact, increases in the proportion of women using injectables between 1995 and 2002 were greatest among Hispanic and non-Hispanic black women; ${ }^{q}$ we found that black women do face an elevated risk of failure relative to non-Hispanic white and other users of contraception when considering all methods combined. However, we did not have a sufficient number of observations to examine whether this difference holds among users of injectables only.

An increase was identified in the risk of failure among users of fertility-awareness-based methods (although not statistically significant): the already very high risk of failure among users of this group of methods in 1995 (22.6\%) rose to $25.3 \%$ by 2002 . On the other hand, the estimate of failure for withdrawal appears to have dropped dramatically — from $28.4 \%$ in 1995 to $18.4 \%$ in 2002 - but the wide confidence intervals surrounding these estimates (a result of the small size of the group using this method) mean that this decrease is not statistically significant. Estimates of the risk of failure for the pill (8.8\% in 1995 and $8.7 \%$ in 2002) and the condom (17.8\% in 1995 and $17.4 \%$ in 2002), the two most widely used reversible contraceptive methods, remained relatively constant from 1995 to 2002.

In 2002, women younger than 30, black women, those who intend a birth in the future, those who already have a birth, those who are poor or near poor, and women in a cohabiting union had a higher risk of failure compared to the relevant reference groups. Examining trends in contraceptive failure among subgroups of women, we found only that those who had never given birth were significantly less likely to become pregnant while using a method in 2002 than in 1995.

The socioeconomic factors associated with differential risks of failure are not the same for all methods. Women living in poverty who rely on a partner-dependent method - the condom or withdrawal - are nearly twice as likely to experience a failure as other women, but those in poverty who use the pill experience the same risk as higher income women who use that method. And while black women who use the condom are more likely to experience a failure than are women from other race/ethnic groups, race/ethnicity has no effect on the risk of failure for the pill or withdrawal.

A woman's relationship with her partner also has differing effects on her risk of failure depending on her contraceptive method. For the pill, formal marriage is associated with the lowest risk of failure, with both those who are not in union and cohabiting women experiencing a higher relative risk. For the condom, however, only cohabiting women face an elevated risk of failure; women who are not in union are no more or less likely to experience a condom failure than married women. And for withdrawal, the critical distinction is between women who have ever been married and those who have not, with the latter experiencing a greater risk

qAuthors' tabulations from the 1995 and 2002 NSFGs. 
of failure. These method-specific differences likely represent the way in which effectiveness in using a method can be affected by the woman's relationship with her sexual partner or partners as well as frequency of intercourse. Pill users who are not in a union may have less frequent intercourse than married pill users, but they may also be more likely to miss pills and delay refills. Similarly, we may see no difference in the risk of failure between single and married condom users if the coital frequency of the former is much less. And for users of the withdrawal method - which is very much a partner dependent method - the woman's risk of failure may be strongly influenced by the degree to which she and her partner have discussed their childbearing intentions, a discussion that is far more likely to have occurred among ever married couples than those who have never been married.

These analyses also indicate that some factors identified as affecting the risk of failure among users of any reversible method have similar effects on the risk of failure among users of the three specific methods that could be examined (pill, condom and withdrawal): the woman's age, her intention toward a future birth, and whether she has already had a birth. These individual method analyses support the conclusion that, for users of all three of these methods and probably for users of every reversible method, women age 30 and older enjoy a much lower risk of failure than younger women, as do contraceptive users who intend no more births and those who have never had a birth.

Findings from other research suggest that the relatively high probabilities of contraceptive failure measured in the current study are plausible. A national study of patterns of contraceptive use among women at risk of unintended pregnancy found that $24 \%$ switch methods during a one-year period, increasing the risk of failure given adjustment to use of a new method [17]. Another study demonstrated the positive relationship between strength of motivation to prevent an unintended pregnancy, and using contraception continuously [18]. A similar positive relationship is found between motivation to prevent unintended pregnancy and greater consistency in using methods (Jennifer Frost, personal communication, May 2007). These findings provide support for the differentials in risk of failure that we found for union status and intentions regarding future births.

In 2002 , more than $98 \%$ of U.S. women 15-44 years of age who had ever had sexual intercourse with a male had used at least one contraceptive method [16]. Ninety percent of all women ages 15-44 had ever had a partner who used the condom and $82 \%$ had ever used the pill [16]. Thus, most American women are experienced at trying to avoid unintended pregnancy. However, while the rate of unintended pregnancy has remained unchanged from 1995 to 2002, there has been a small decline in the proportion of unintended pregnancies that occurred during use of contraception (51\% in 1994 v. 48\% in 2001) [1], and overall use of contraception among women at risk of unintended pregnancy has also dropped, from $92.5 \%$ in 1995 to $89.3 \%$ in $2002 .{ }^{\mathrm{r}}$ Both of these trends may indicate an increased tendency among women to rely on chance rather than contraception, particularly among those subgroups most vulnerable to the risk of failure. In fact, the proportion using contraception among women in poverty at risk of pregnancy dropped from $92.1 \%$ in 1995 to $86.3 \%$ in $2002 .{ }^{\mathrm{s}}$

Barriers to effective contraceptive use include partner opposition, difficulty in obtaining a method, and difficulty in use. But even among women who overcome those hurdles and do use a method, the effectiveness of a method greatly depends on vigilance and effective use by the woman and her partner. The substantial differences in probabilities of failure among socioeconomic subgroups in the United States indicates that many of these difficulties are not being overcome, and they may have become even harder to overcome in recent years for some

r Ibid.

SIbid. 
socially and economically disadvantaged groups of women than for other women. The extent to which relatively high probabilities of failure may lead to discouragement among users and lessened use is not yet known. But high risks of failure could make it more difficult to convince nonusers to adopt contraception and to convince those already using to continue.

Particular attention should be paid to reducing barriers to contraceptive access for disadvantaged groups of women. In addition, there is much room to improve effectiveness of condom use given the large gap between the probabilities of failure during typical and perfect use. The Healthy People 2010 goal of reducing the overall probability of contraceptive failure from $13 \%$ in 1995 to $7 \%$ by 2010 , as well as reducing failure among specific disadvantaged population groups, will be very difficult in the absence of major policy and programmatic interventions. A number of strategies need to be pursued simultaneously if this goal is to be reached: increased education and provision of information to reduce misperceptions about methods; improved access to contraceptive services including a wide choice of methods including long-acting methods such as injectables, implants and IUDs; and more widespread practice of counseling for both women and men to improve consistency and correctness of use, as well as to improve communication between partners about contraceptive use and planning pregnancies.

\section{References}

1. Finer LB, Henshaw SK. Disparities in rates of unintended pregnancy in the United States, 1994 and 2001. Perspect Sex Reprod Health 2006;38:90-96. [PubMed: 16772190]

2. Fu H, Darroch JE, Haas T, Ranjit N. Contraceptive failure rates: new estimates from the 1995 National Survey of Family Growth. Fam Plann Perspect 1999;31:56-63. [PubMed: 10224543]

3. Trussell J, Vaughan B. Contraceptive failure, method-related discontinuation and resumption of use: results from the 1995 National Survey of Family Growth. Fam Plann Perspect 1999;31:64-72. 93. [PubMed: 10224544]

4. Ranjit N, Bankole A, Darroch JE, Singh S. Contraceptive failure in the first two years of use: differences across socioeconomic subgroups. Fam Plann Perspect 2001;33:19-27. [PubMed: 11271541]

5. U.S. Department of Health and Human Services. Washington, DC: U.S. Government Printing Office; 2000 Nov. Healthy People 2010, 2nd ed. With understanding and improving health and objectives for improving health 2 vols.

6. Jones EF, Forrest JD. Contraceptive failure rates based on the 1988 NSFG. Fam Plann Perspect 1992;24:12-19. [PubMed: 1601121]

7. Finer LB, Henshaw SK. Abortion incidence and services in the United States in 2000. Perspect Sex Reprod Health 2003;35:6-15. [PubMed: 12602752]

8. Jones RK, Kost K. Underreporting of induced and spontaneous abortion in the United States: an analysis of the 2002 National Survey of Family Growth. Stud Fam Plann 2007;38:187-197. [PubMed: 17933292]

9. Jones RK, Darroch JE, Henshaw SK. Contraceptive use among U.S. women having abortions in 20002001. Perspect Sex Reprod Health 2002;34(6):294-303. [PubMed: 12558092]

10. Fu H, Darroch JE, Henshaw SK, Kolb E. Measuring the extent of abortion underreporting in the 1995 National Survey of Family Growth. Fam Plann Perspect 1998;30:128-133. 138. [PubMed: 9635261]

11. Jones EF, Forrest JD. Underreporting of abortion in surveys of U.S. women: 1976 to 1988. Demography 1992;29:113-125. [PubMed: 1547898]

12. Vaughan B, Trussell J, Menken J, Jones EF, Grady W. Contraceptive efficacy among married women aged 15-44 years. Vital Health Stat 1980;23:1-48.

13. Peto R, Pike MC, Armitage P, et al. Design and analysis of randomized clinical trials requiring prolonged observation of each patient. II. analysis and examples. British J Cancer 1977;35:1-39.

14. SAS Institute Inc. SAS, computer programming package for statistical analysis. 2007 
15. Lepkowski JM, Mosher WD, Davis KE, Groves RM, van Hoewyk J, Willem J. National Survey of Family Growth, Cycle 6: Sample design, weighting, imputation, and variance estimation. National Center for Health Statistics, Vital Health Stat 2006; Series 2(No 142):92. pages.

16. Mosher, WD.; Martinez, GM.; Chandra, A.; Abma, JC.; Wilson, SJ. Advance data from vital and health statistics; no. 350. Hyattsville, Maryland: National Center for Health Statistics; 2004. Use of contraception and use of family planning services in the United States: 1982-2002.

17. Frost JJ, Singh S, Finer LB. Women's one-year contraceptive use patterns, 2004. Perspect Sex Reprod Health 2007;39:48-55. [PubMed: 17355381]

18. Frost JJ, Singh S, Finer LB. Factors associated with contraceptive use and nonuse, United States, 2004. Perspect Sex Reprod Health 2007;39 90-q9.

19. Trussell J, Grummer-Strawn L, Rodriguez G, Van Landingham M. Trends and differentials in breastfeeding behavior: evidence from the WFS and DHS. Population Studies 1992;46:285-307.

20. Hatcher, RA.; Trussell, J.; Nelson, AL.; Cates, W.; Stewart, FH.; Kowal, D. Contraceptive technology. 19th edition. New York, NY: Ardent Media; 2007.

\section{Appendix}

\section{Appendix: Segment Data}

A segment is defined as an uninterrupted period of use of a particular contraceptive method.

Segments have varying lengths and are characterized by the method used and the duration of use in months. The segment also contains information on the events that occurred at the termination of the segment, such as a pregnancy, a switch to another method, a switch to nonuse, or censoring by the interview. In addition, segments carry information on characteristics of the user such as her age at the start of the interval and her race and ethnicity. Separate segments are identified for characteristics that can change, such as the woman's marital and cohabitation status at the start of the interval of use. Some women contribute more than one segment to the analysis if they stopped use of a method within the observation window and then began another segment of use before their interview. Also, in analyses that include union status, women will contribute multiple segments if their marital or cohabitation status changes during a continuous interval of method use.

Most segments observed during the calendar period began after January 1999, but some were left-truncated; they began before and were observed in progress at the start of the observation window. Because the starting date of these segments is known, they are included in the data, but only those months of use observed after January 1999 are included in our analyses.

Similarly, some segments are "right censored" by the date of interview, when observation ends. The resulting data set used in our analyses contains 9,569 segments of contraceptive use (reversible methods only).

In the interview for the NSFG survey, the woman's contraceptive history was obtained after the pregnancy history was recorded in the calendar. In this way, pregnancies served to help in the recall of periods of contraceptive use. We identify contraceptive failures in the NSFG by examining whether contraception was used in the same month as a conception. If the woman reports having stopped method use in the same month as the conception, we examine the response to the question, "Before you became pregnant, had you stopped using all methods of birth control?" If the answer was "no", we recorded a failure.

If method use continued into months following the conception, a failure is assigned to the segment of use, unless use continues beyond four months after the conception. The NSFG questionnaire assumes that these are failures, and does not ask the woman if she had stopped using, even though it seems unlikely that someone would continue to use contraception throughout a pregnancy. For these cases, we check additional information from the survey to determine if the woman had actually stopped use earlier. In the period covered by our analysis, 
there were 52 cases of condom use that continued for more than four months after conception. Of these 42 were determined to be actual failures, while the others were determined based on other information in the survey to be nonusers of contraception at the time of conception. Of the 29 pill users and the 62 users of other methods whose use continued for more than 4 months after conception, 23 and 19, respectively, were determined to be failures.

For each segment, we also calculated "exposure" to the risk of pregnancy during contraceptive use. The calendar survey records the months in which contraception was used but not exactly how long the method was used. For example, a woman's calendar may indicate pill use in March, April and May. This means she began use sometime in March and ended use sometime in May. We assume that, on average, use begins and ends in the middle of the month (e.g. some women start early in the month, some start later). We also assume that if a new method is recorded in the next month (June, in the above example) that use of the new method begins mid-month so that use of the first method ends when the new method begins (i.e., a method switch in June). In the above example, this means that exposure for the pill would be $1 / 2$ month for March, 1 month for April, 1 month for May, and $1 / 2$ month for June, with a total exposure equal to 3 months.

On the other hand, if a segment of use is not followed by another method but instead by nonuse, total exposure is calculated as total number of months of use minus one; or, as in the example above, $1 / 2$ month for March, 1 month for April, and $1 / 2$ month for May, with a total exposure equal to 2 months.

Exposure for segments in which use begins and ends in the same month was assigned to be $1 / 3$ month. This is based on the calculations shown in the appendix to Trussell et al. (1992), which demonstrates the appropriateness of this assumption [19].

If a woman began using a method before the start of the calendar in January 1999, say, 3 months prior to January and continued use for the first 4 months of 1999, we include only the 4 months observed in 1999 in her exposure calculation (and she enters the life tables at duration 4, not zero). She would receive a full month of exposure in January, even though that is her first month of use in the calendar because she is observed to be using for the entire month.

Some women who were using contraception at interview may not have been exposed to the risk of pregnancy if they were in fact already pregnant but did not yet realize it. To prevent inclusion of this portion of their segments of use, the exposure for method segments that continue up to the month of interview were censored two months prior to interview. By examining the proportion of currently pregnant women in each week of pregnancy, we found that the proportion in their $7^{\text {th }}$ week of pregnancy was similar, or even higher, than the proportion at subsequent weeks of pregnancy. It would be expected to be higher because some will lose the pregnancy or end it. When we limit the observation of method segments to 2 months prior to interview, we eliminate the inclusion of additional months of exposure for women who might be 6 weeks pregnant or less but do not yet know it. The date of conception in the NSFG was calculated as date of interview minus number of weeks pregnant (as reported by the woman). Tabulations of data indicate that pregnancies of duration $0-2$ weeks correspond to a conception month equal to the interview month, 3-6 is equal to one month prior to interview, and 7-10 weeks is equal to 2 months prior to interview. So those women who might still be pregnant even after our censoring would have to be at least 7 weeks pregnant, or, if they reported in months, two months pregnant.

For segments censored by interview, we also added $1 / 2$ month of exposure to the total exposure calculated. Without the additional half-month, we would have only $1 / 2$ month of exposure in the denominator of our failure calculations but a full month's worth of events in the numerator. The reason is that we do not censor the failures that occur two months prior to interview. 
However, recall that we only include $1 / 2$ month of exposure in the last month observed for a segment. If we do not add $1 / 2$ month to the final segment observed prior to interview we would overestimate the failure rates by excluding $1 / 2$ month of exposure yet including all failures that occurred in a full month.

Respondents in the NSFG were allowed to report more than one method in each month of their calendar histories of contraceptive use. In fact, numerous respondents did so; $15.8 \%$ of all segments had more than one method listed as having been used. About two-thirds of multiple method use indicates the use of a "backup" method, such as condom use among pill users. Other multiple method use indicates that the woman alternates between methods, such as the use of withdrawal and condom. In cases where one of the multiple methods used was a highly effective or hormonal method - injectables, implants, IUD, or the pill — we assigned the more effective method to the segment, using the priority ranking of effectiveness given in Contraceptive Technology [20]. For cases of multiple method use among the remaining methods not involving these effective or hormonal methods, we also assigned to the segment the most effective of the methods used based on the prioritized ranking of effectiveness if the respondent indicated that the methods had been used simultaneously. If instead the respondent indicated that she had alternated use of the methods, we coded the method for these segments as "sequential use of methods less effective than the pill" and these segments are classified as "Other". Segments of sterilization - either the woman's or her partner's — were excluded from the analysis.

The segment data set used for analysis consisted of 9,033 intervals of contraceptive use, including 715 segments of injectables, 2,541 segments of the pill, 3,845 segments of the male condom, 848 segments of withdrawal, 236 segments of fertility-awareness-based methods, 67 segments of spermicides, and 781 segments of other methods, including the IUD, implants, the contraceptive patch, injectable methods no longer used in the U.S., cervical cap, diaphragm, contraceptive film, and all sequential use of methods less effective than the pill. As indicated above, the total number of segments for each method in analyses that include union status is higher than these numbers because individual segments are broken into two or more segments if and when the union status changes. Thus, there were an additional 536 segments $(9,569-$ 9,033 ) in analyses with union status included. Any change in union status during a period of continuous contraceptive use results in a new segment; however, the new segment begins at the next month of use. For example, if a woman begins using the pill within the observation period and her union status changes from cohabiting to married in her fifth month of use, the first segment is characterized as a cohabiting segment ending at the completion of 4 months, and the second segment is characterized as a married segment commencing at the $5^{\text {th }}$ month of use. 


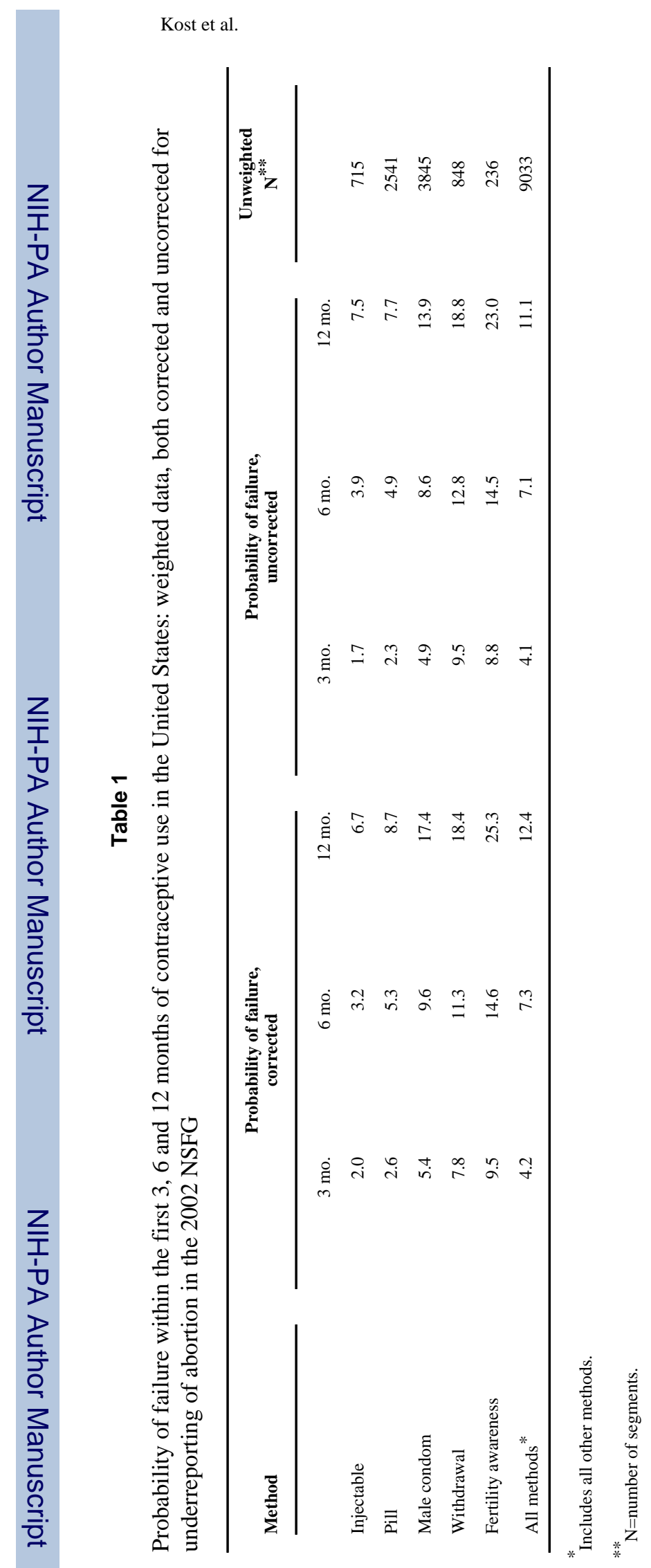

Contraception. Author manuscript; available in PMC 2010 January 26. 


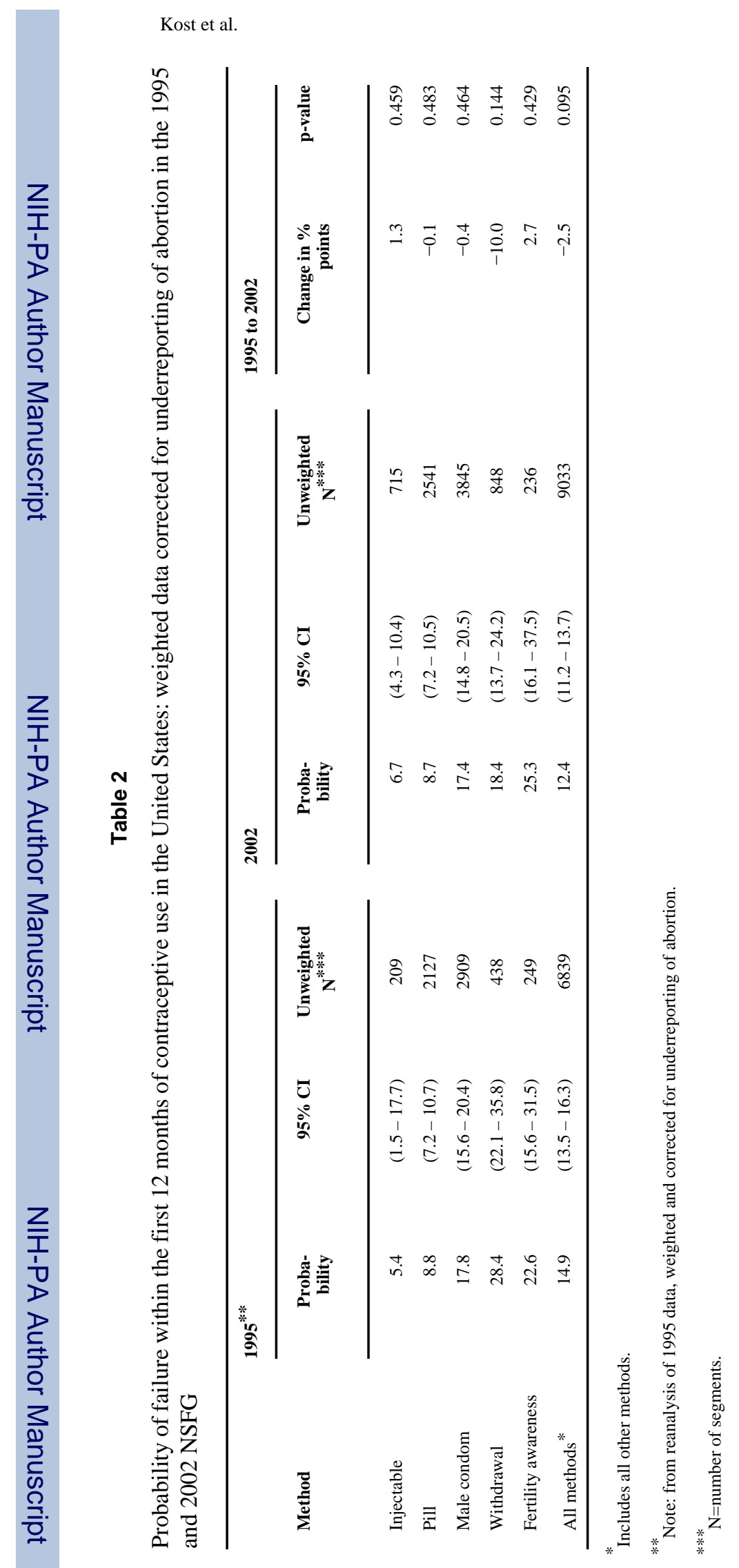

Contraception. Author manuscript; available in PMC 2010 January 26. 


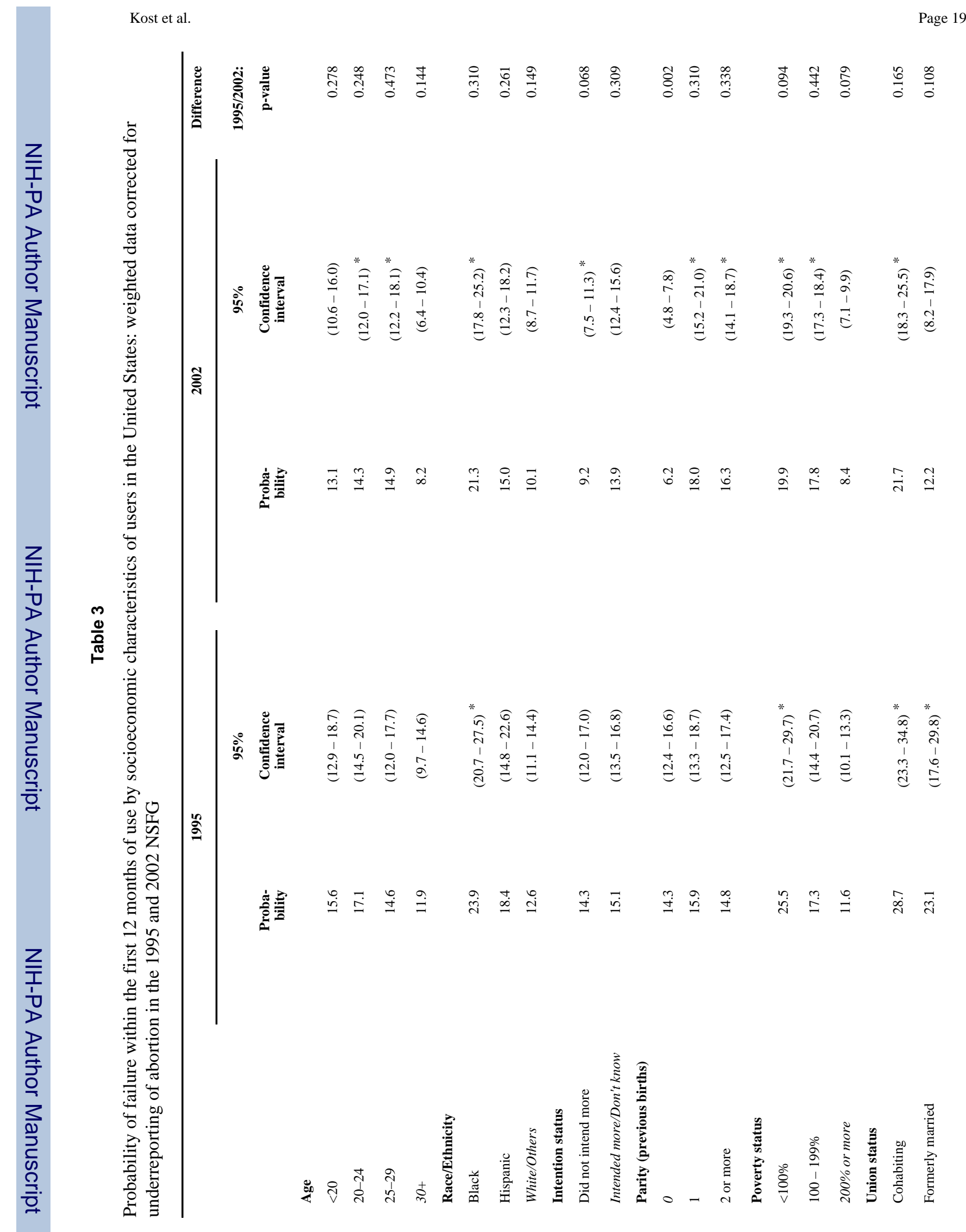

Contraception. Author manuscript; available in PMC 2010 January 26. 


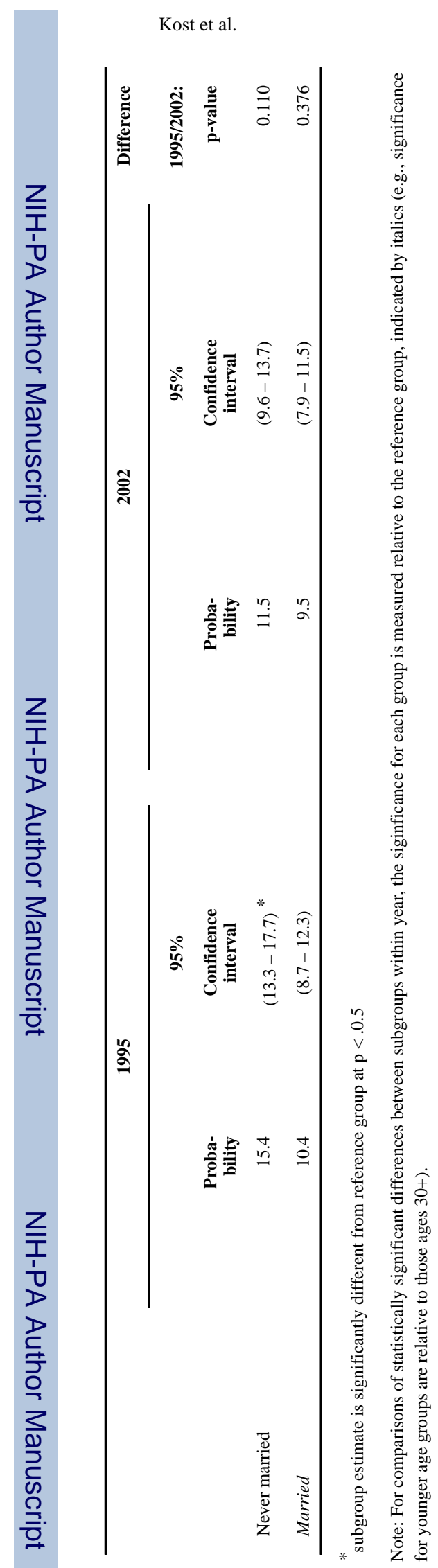

Page 20

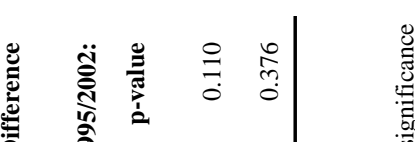


Table 4

Relative risk of failure within the first 12 months of contraceptive use by socioeconomic characteristics of users of the pill, condom, and withdrawal in the United States: unweighted data corrected for underreporting of abortion in the 2002 NSFG

\begin{tabular}{|c|c|c|c|}
\hline Pill users & $\begin{array}{c}\text { Relative } \\
\text { risk* }\end{array}$ & p-value & 95\% C.I. \\
\hline \multicolumn{4}{|l|}{ Age (30+) } \\
\hline $20-29$ & 1.67 & 0.0498 & $(1.33-4.28)$ \\
\hline$<20$ & 2.38 & 0.0036 & $(1.00-2.80)$ \\
\hline \multicolumn{4}{|l|}{ Intention status (no more) } \\
\hline Intend more or don't know & 1.73 & 0.0081 & $(1.15-2.60)$ \\
\hline \multicolumn{4}{|l|}{ Union status (currently married) } \\
\hline Cohabiting & 2.54 & $<.0001$ & $(1.63-3.98)$ \\
\hline Not in union & 1.93 & 0.0025 & $(1.26-2.95)$ \\
\hline \multicolumn{4}{|l|}{ Parity (no births) } \\
\hline 1 or more births & 7.94 & $<.0001$ & $(4.99-12.64)$ \\
\hline \multicolumn{4}{|l|}{ Condom users } \\
\hline \multicolumn{4}{|l|}{ Age (30+) } \\
\hline$<30$ & 1.55 & 0.0093 & $(1.11-2.16)$ \\
\hline \multicolumn{4}{|l|}{ Intention status (no more) } \\
\hline Intend more or don't know & 1.47 & 0.0101 & $(1.10-1.97)$ \\
\hline \multicolumn{4}{|l|}{ Union status (not cohabiting) } \\
\hline Cohabiting & 1.61 & 0.0009 & $(1.22-2.13)$ \\
\hline \multicolumn{4}{|l|}{ Parity (no births) } \\
\hline 1 or more births & 2.54 & $<.0001$ & $(1.88-3.43)$ \\
\hline \multicolumn{4}{|l|}{ Race/Ethnicity (all other) } \\
\hline Nonhispanic black & 1.63 & 0.0001 & $(1.27-2.09)$ \\
\hline \multicolumn{4}{|l|}{ Poverty status (200\% or more) } \\
\hline Less than $200 \%$ & 1.91 & $<.0001$ & $(1.46-2.49)$ \\
\hline \multicolumn{4}{|l|}{ Withdrawal users } \\
\hline \multicolumn{4}{|l|}{ Intention status (no more) } \\
\hline Intend more or don't know & 3.12 & 0.0002 & $(1.72-5.63)$ \\
\hline \multicolumn{4}{|l|}{ Union status (ever married) } \\
\hline Never married & 2.25 & 0.0005 & $(1.42-3.56)$ \\
\hline \multicolumn{4}{|l|}{ Parity (no births) } \\
\hline 1 or more births & 4.19 & $<.0001$ & $(2.31-7.60)$ \\
\hline \multicolumn{4}{|l|}{ Poverty status ( $200 \%$ or more) } \\
\hline Less than $200 \%$ & 1.94 & 0.0036 & $(1.24-3.02)$ \\
\hline
\end{tabular}

* Risk relative to that for the reference group, indicated in parentheses. 